13. Heusser, H., Engel, C., Herzig, P. T. and Plattner, P. A. Helv. Chim. Acta 23 (1950) 2229.

14. Engel, C. J. Am. Chem. Soc. 78 (1956) 4727.

15. Venus-Danilowa, E. D. J. Gen. Chem. USSR 11 (1941) 847; Chem, Abstr. 36 (1942) 4094.

16. Aston, J. G. and Greenburg, R. B. J. Am. Chem. Soc. 62 (1940) 2590.

17. Morrell, R. S. and Bellars, A. E. J. Chem. Soc. 85 (1904) 345.

18. Rappe, C. Arkiv Kemi 21 (1963) 503.

Received December 6, 1963.

Crystallographic Data on $\mathbf{N}, \mathbf{N}, \mathrm{N}^{\prime}, \mathrm{N}^{\prime}$ Tetrakis (2-aminoethyl) ethylenediamine Pentahydrochloride $=$ [Penten,5HCl], Hydroxy-PentenCobalt(III) Iodide and HydroxyPenten-Cobalt(III) Perchlorate

CARL OLAF HA A ENSEN

Department of Inorganic Chemistry, University of Aarhus, Aarhus C, Denmark

Causs, Moser and Schwarzenbach ${ }^{1}$ preGpared "penten", 5HCl in connection with investigations of metal complexes with polyamines. Crystallographic data on this compound (1) and on the cobalt(III) complexes $[\mathrm{Co}(\mathrm{OH})$ penten $] \mathrm{I}_{2} \quad(2)$ and $[\mathrm{Co}(\mathrm{OH})$ penten $]\left(\mathrm{ClO}_{4}\right)_{2} \quad(3)$ were obtained from rotation and Weissenberg photographs recorded with $\mathrm{Cu} K a$ radiation and from precession photographs recorded with
$\mathrm{Cu} K a$ and $\mathrm{MoK} \alpha$ radiation. The densities of the crystals were measured by the flotation method.

The crystals of all three compounds are monoclinic; from systematic absences (1) and (2) must have the space group $P 2_{1} / c$ (No. 14, $C_{2 h}{ }^{5}$ ), although (3) has space group $P 2$ (No. 3, $C_{2}{ }^{1}$ ) or $P m$ (No. 6, $C_{s}{ }^{1}$ ) the reflections $h 0 l$ with $l=2 n+1$ and $0 k 0$ with $k=2 n+1$ are very weak and so (2) and (3) are nearly isomorphous.

Detailed studies of these three compounds are in progress.

The crystals were kindly supplied by professor G. Schwarzenbach, Zürich.

1. Gauss, W., Moser, P. and Sehwarzenbach, G. Helv. Chim. Acta. 35 (1952) 2359.

Received December 9, 1963.

\section{A Conversion of D-Glucose to D-Mannose under Acidic Conditions}

PER JER K E M A N

Träkemiska Avdelningen, Svenska Träforskningsinstitutet, Stockholm, Sweden

In an attempt to prepare D-manno1 furanose pentaacetate, 2,3:5,6-di- $O$-isopropylidene-D-mannose was treated with a mixture of acetic acid, acetic anhydride and sulphuric acid at room temperature. When the deacetylated product was investigated by paper chromatography, how ever, it gave two spots, which corresponded to D-glucose and D-mannose. By fractionation of the mixture these two sugars were

Table 1.

(1)

Formula

Formula wt.

$D_{m}\left(\mathrm{~g} \cdot \mathrm{ml}^{-1}\right)$

$D_{x}\left(\mathrm{~g} \cdot \mathrm{ml}^{-1}\right)$

Space group

$Z$ (calc.)

$a(\AA)$

$b \quad(\AA)$

$c(\AA)$

$\beta\left({ }^{\circ}\right)$

V $\left(\AA^{3}\right)$

$\mathrm{C}_{10} \mathrm{H}_{33} \mathrm{~N}_{6} \mathrm{Cl}_{5}$
414.7
1.34
1.35
$P 2_{1} / c$
4
10.19
23.18
9.96
120.0
2037

(2)

$\mathrm{CoC}_{10} \mathrm{H}_{29} \mathrm{~N}_{6} \mathrm{OI}_{2}$
562.2
2.07
2.07
$P 2 / c$
4
10.52
15.31
11.31
98.4
1802

(3)

$\mathrm{CoC}_{10} \mathrm{H}_{29} \mathrm{~N}_{6} \mathrm{O}_{9} \mathrm{Cl}_{2}$
507.2
1.71
1.71
4
10.57
15.99
11.76
98.4
1966

Acta Chem. Scand. 17 (1963) No. 10 
isolated in pure states in the relative proportions 8:2.

Rearrangements of cyclitol acetates and acetylated carbohydrates under acidic conditions have recently been reported in the literature. Fletcher and co-workers studied the epimerisation of cyclitol ${ }^{1}$ and 1,5-anhydro-glycitol ${ }^{2}$ acetates in liquid hydrogen fluoride. Angyal and co-workers ${ }^{3}$ studied the epimerisation of cyclitol acetates in $95 \%$ acetic acid containing $1.5 \%$ sulphuric acid at $100^{\circ}$. In these reactions a system containing three adjacent $O$-acetyls is rearranged from the cis-trans (I) to the trans-cis (IV) configuration. When no deacetylation takes place, the reaction is reversible. According to the proposed mechanism, ${ }^{2,3}$ a seven-membered ring (II) is formed between the two $O$-acetyl groups in the cis-position. This ring is then opened, with inversion, by attack of the $O$-acetyl group in the trans-position, with formation of a five-membered ring (III). The epimerisation of acylated sugars in liquid hydrogen fluoride, ${ }^{4}, 5$ of $\mathrm{D}$-quinic acid in $95 \%$ acetic acid containing $1.5 \%$ sulphuric acid ${ }^{6}$ and of acetylated 6-deoxy6-iodo-aldehydo-sugars in acetic anhydride and zinc chloride ${ }^{7}$ are related reactions. It is probable that the conversion of lactose octaacetate to a neolactose derivative on treatment with phosphorus pentachloride and aluminium chloride and other conversions studied by Hudson and Richtmyer ${ }^{8}$ also proceed by a similar mechanism. ${ }^{2}$

The epimerisation of the D-mannofuranose derivative mentioned above is obviously another example of the same re-

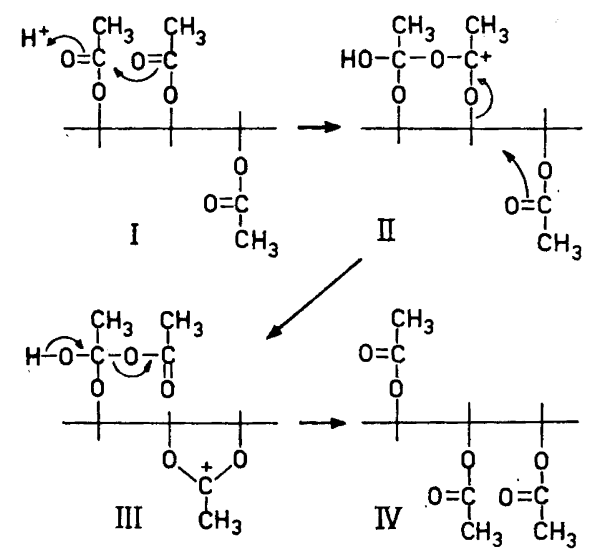

arrangement, as any cyclic sugar acetate, in one of its anomeric forms, should have the cis-trans or trans-cis arrangement at the three $O$-acetyls at $\mathrm{C}-1, \mathrm{C}-2$, and C-3. The acetolysis of a number of furanosidic and pyranosidic sugar derivatives was therefore investigated under the same conditions (Table 1). In all examples the final product was a mixture of fully acetylated sugars, which was deacetylated and studied by paper chromatography. As can be seen from Table 1 , no rearrangement was observed for the pyranosides, ir: agreement $w^{\text {th }}$ numerous previous observations that acetolysis and anomerisation are not accompanied under these conditions by epimerisation. The three $D$-mannofuranose derivatives all yielded about $80 \%$ of $\mathrm{D}$-glucose. The phenyl. $\beta$-Dglucofuranoside yielded a trace of $\mathrm{D}$-mannose but apart from that no epimerisation was observed for the other furanosides studied.

Acetolysis of furanosidic isopropylidene derivatives of $\mathrm{D}$-glucose and $\mathrm{D}$-xylose has been used as a preparative method for the synthesis of their furanosidic acetates. ${ }^{9},{ }^{10}$ Under these conditions little or no conversion into pyranosidic acetates was observed. A chromatographic investigation of the product from the acetolysis of 2,3:5,6-di- $O$-isopropylidene-D-mannose also revealed that no pyranosidic acetates had been formed.

It is easily understood why the furanosidic acetates should be rearranged more readily than those of the pyranosides. The formation of the seven-membered ring (II), the attack from behind by the trans-O. acetyl group and the formation of the five-membered ring (III) should all be facilitated in the furanose form but not in the pyranose. Only one of the anomeric acetates, the one with the cis-trans- or trans-cis arrangement at the three $O$-acetyls at $\mathrm{C}-1, \mathrm{C}-2$, and $\mathrm{C}-3$, can be rearranged. The stability of the furanosidic acetates of D-glucose, D-galactose, D-xylose and $\mathrm{L}_{\mathrm{H}}$-arabinose, compared to that of D-mannose, could be due to the low concentration of this anomer in the equilibrium mixture of the former acetates. Nothing is known, however, about these equilibria. Another possible reason for the above results is that all the sugars investig. ated, except $\mathrm{D}$-mannose, had the transarrangement at $\mathrm{C}-2$ and $\mathrm{C}-3$ and represent the energetically-favoured epimer. It is probable that the furanosidic acetates of their epimers, e.g. ribose, lyxose and 
Table 1. Reaction of sugar derivatives with acetic acid, acetic anhydride and sulphuric acid.

2,3:5,6-Di- $O$-isopropylidene-D-mannose

1,5,6-Tri- $O$-acetyl-2,3-O-isopropylidene-D-mannose

Methyl $\beta$-D-mannofuranoside

Phenyl $\beta$-D-glucofuranoside

3,5,6-Tri-O-acetyl-1,2-O-isopropylidene-D-glucose

Phenyl $\beta$-D-galactofuranoside

Phenyl $\beta$-D-xylofuranoside

Phenyl $\alpha$-L-arabinofuranoside

$2,3,4,6$-Tri- $O$-acetyl-ethyl- $\beta$-D-mannoside

Methyl $\alpha$-D-mannopyranoside

Methyl $\beta$-D-glucopyranoside

talose, would undergo the same rearrangement.

Experimental. The sugar derivative ( $1 \mathrm{~g})$ was dissolved in a mixture of glacial acetic acid $(30 \mathrm{ml})$ and acetic anhydride $(4 \mathrm{ml})$. Concentrated sulphuric acid $(1.5 \mathrm{ml})$ was added with stirring and external cooling with ice. The solution was kept for $60 \mathrm{~h}$ at room temperature and then poured into ice-water $(100 \mathrm{ml})$. The aqueous solution was shaken with chloroform $(2 \times 20 \mathrm{ml})$ and the chloroform solution was washed successively with water, saturated aqueous sodium hydrogen carbonate solution and water. It was then concentrated to a syrup. This syrup, which contained the corresponding sugar acetates, was deacetylated 11 by dissolving it in $10 \mathrm{ml}$ water and $80 \mathrm{ml}$ methanol and treating with $15 \mathrm{ml} 45 \%$ aqueous trimethylamine. The solution was kept overnight and then concentrated under reduced pressure. The resulting syrup was investigated by paper chromatography, using ethyl acetate-pyridinewater, 8:2:1, and Whatman No. 1 filter paper. D-Glucose, m.p. $143-5^{\circ}$ and D-mannose, m.p. $128-31^{\circ}$ were isolated from the reaction mixture obtained from $2,3: 5,6$-di- $O$-isopropylidene-D-mannose by chromatographic separation on Whatman No. 3 filter paper in the same solvent mixture. The ruantitative determinations were made according to Saeman et al. ${ }^{12}$ and chromatography of the acetates on dimethyl sulphoxide-impregnated paper in isopropyl ether was according to Wickberg. ${ }^{13}$
Product after deacetylation $80.5 \%$ D-glucose, $19.5 \%$ D-mannose

$80.1 \%$ D glucose, $19.9 \%$ D-mannose $73.0 \%$ D-glucose, $27.0 \%$ D-mannose D-glucose, traces of D-mannose D-glucose D.galactose D-xylose L-arabinose D-mannose D-mannose D-glucose

Acknowledgement. The author is indebted to Professor Bengt Lindberg for his interest and helpful advice and to Mr Leif Jacobsson for his skilful assistance.

1. Hedgley, E. J. and Fletcher, Jr., H. G. J. Am. Chem. Soc. 84 (1962) 3726.

2. Hedgley, E. J. and Fletcher, Jr., H. G. J. Am. Chem. Soc. 85 (1963) 1615.

3. Angyal, S. J., Gorin, P. A. J. and Pitman, M. Proc. Chem. Soc. 1962337.

4. Pedersen, C. and Fletcher, Jr., H. G. J. Am. Chem. Soc. 82 (1960) 945.

5. Pedersen, C. Acta Chem. Scand. 16 (1962) 1831.

6. Gorin, P. A. J. Can. J. Chem. 41 (1963) 2417.

7. Micheel, F. and Böhm, R. Tetrahedron Letters 1962107.

8. Richtmyer, N. K. Advan. Carbohydrate Chem. 1 (1945) 37.

9. Börjeson, H., Jerkeman, P. and Lindberg, B. Acta Chem. Scand. 17 (1963) 1705.

10. Jerkeman, P. and Lindberg, B. Acta Chem. Scand. 17 (1963) 1709.

11. Whelan, W. J. Private communication.

12. Saeman, J. F., Moore, W. E., Mitchell, R. L. and Millet, M. A. Tappi 38 (1954) 336.

13. Wickberg, B. Methods in Carbohydrate Chem. Vol. 1 (1962) 31.

Received December 7, 1963. 